\title{
Enhanced Piezoelectric Behavior of PVDF Nanocomposite by AC Dielectrophoresis Alignment of ZnO Nanowires
}

\author{
Kyungwho Choi, ${ }^{1}$ Woongchul Choi, ${ }^{2}$ Choongho Yu, ${ }^{2,3}$ and Yong Tae Park ${ }^{4}$ \\ ${ }^{1}$ New Transportation Systems Research Center, Korea Railroad Research Institute, Uiwang-si, Gyeonggi-do 16105, Republic of Korea \\ ${ }^{2}$ Department of Materials Science and Engineering, Texas A\&M University, College Station, TX 77843, USA \\ ${ }^{3}$ Department of Mechanical Engineering, Texas A\&M University, College Station, TX 77843, USA \\ ${ }^{4}$ Department of Mechanical Engineering, Myongji University, Yongin-si, Gyeonggi-do 17058, Republic of Korea
}

Correspondence should be addressed to Choongho Yu; chyu@tamu.edu and Yong Tae Park; ytpark@mju.ac.kr

Received 10 April 2017; Revised 14 August 2017; Accepted 12 September 2017; Published 15 October 2017

Academic Editor: Magnus Willander

Copyright (c) 2017 Kyungwho Choi et al. This is an open access article distributed under the Creative Commons Attribution License, which permits unrestricted use, distribution, and reproduction in any medium, provided the original work is properly cited.

\begin{abstract}
In contrast to commercial piezoelectric ceramics, lead-free materials such as $\mathrm{ZnO}$ and a polymer matrix are proper candidates for use in ecofriendly applications. In this article, the authors represent a technique using $\mathrm{ZnO}$ nanowires with a polyvinylidene fluoride (PVDF) matrix in a piezoelectric polymer composite. By aligning the nanowires in the matrix in a desired direction by AC dielectrophoresis, the piezoelectric behavior was enhanced. The dielectric constant of the composite was improved by increasing the concentration of the $\mathrm{ZnO}$ nanowires as well. Specifically, the resulting dielectric constant shows an improvement of $400 \%$ with aligned $\mathrm{ZnO}$ nanowires by increasing the poling effect compared to that of a randomly oriented nanowire composite without a poling process.
\end{abstract}

\section{Introduction}

Lead zirconate titanate (PZT), which is a typical piezoelectric material, is widely used in the aviation $[1,2]$, automobile $[3,4]$, and precision machinery [5] industries due to its excellent performance capabilities. However, while there have been various studies of lead-free piezoelectric materials, leadbased materials have adverse effects on the environment and on human health [6]. Moreover, piezoelectric ceramics are vulnerable to external vibration or shocks given their brittleness. In order to overcome this, a polymeric material such as polyvinylidene fluoride (PVDF), which is flexible and environmentally friendly, was introduced $[7,8]$. However, ceramic fillers must be incorporated because piezoelectric polymers are not applicable alone due to their low dielectric constant [9]. To obtain higher piezoelectric performance, lead nickel niobate-lead zirconate titanate (PNN-PZT) [10], $\mathrm{BaTiO}_{3}$ [11], lead magnesium niobate-lead titanate (PMNPT) [12], and $\mathrm{ZnO}$ [13-18] can be used as fillers. Among them, $\mathrm{ZnO}$ nanowires have been found to be mechanically strong, biocompatible, and environmentally friendly, making them suitable candidates for wearable electronics or biosensors [19].

In this article, a $\mathrm{ZnO}$ nanowire/PVDF composite with a high dielectric constant was synthesized and piezoelectric behavior was observed while varying the concentration of the $\mathrm{ZnO}$ nanowires, the nanowire alignment process, and the poling condition. The dielectric constant was increased with an increase in the $\mathrm{ZnO}$ nanowire concentration. Specifically, the alignment process of the nanowires enhanced the poling effect due to the directionality of the network structures. The resulting dielectric constant shows an improvement of nearly $400 \%$ when compared to the result with a bare composite film, implying that a well-designed network structure of the composite would be a viable approach to improve the piezoelectric performance.

\section{Experimental}

2.1. $\mathrm{ZnO}$ Nanowire Synthesis. $\mathrm{ZnO}$ nanowires were synthesized by chemical vapor deposition (CVD). Zn foil (SigmaAldrich, purity $99.9 \%$ ) was cut into $1 \mathrm{~cm}$ squares and then 
cleaned with acetone, IPA, and DI water. $5 \mathrm{mM}$ of a $\mathrm{ZnCl}_{2}$ (Fisher Scientific, purity 97.8\%) solution was prepared as a catalyst for the growth of the $\mathrm{ZnO}$ nanowire. The as-prepared catalyst solution was spin coated onto the $\mathrm{Zn}$ foil five times at $2000 \mathrm{rpm}$ for $20 \mathrm{sec}$. Through this step, a $\mathrm{Zn}$ plate could be obtained with uniformly distributed $\mathrm{ZnCl}_{2}$. The as-prepared $\mathrm{Zn}$ foil with the $\mathrm{ZnCl}_{2}$ was then placed in the center of a quartz tube with a diameter of one inch, after which the CVD process was performed. Before the heating process, oxygen gas was applied at $50 \mathrm{sccm}$ for $10 \mathrm{~min}$ in order to remove all other gases in the tube. With the oxygen atmosphere, the oxygen flow rate was decreased to $10 \mathrm{sccm}$ and the furnace was heated to $70^{\circ} \mathrm{C} / \mathrm{min}$ for $10 \mathrm{~min}$. The $\mathrm{Zn}$ foil was allowed to react with oxygen at $700^{\circ} \mathrm{C}$ for $1 \mathrm{hr}$, and the specimen was subsequently collected after natural cooling to room temperature. The $\mathrm{ZnO}$ nanowires were detached from the $\mathrm{Zn}$ foil in IPA solution using a bath type sonicator (1510RMT, Branson) for 30 seconds. Then, the solvent in the $\mathrm{ZnO}$ nanowire-containing solution was evaporated by drying the solution on a glass plate at $70^{\circ} \mathrm{C}$ for $3 \mathrm{hrs}$.

2.2. Nanocomposites Film Fabrication. In order to synthesize the $\mathrm{ZnO} / \mathrm{PVDF}$ composite, the $\mathrm{ZnO}$ nanowires and polyvinylidene fluoride (PVDF, Arkema, Kynar HSV 900) were mixed into a N-methyl-2-pyrrolidone (NMP, J. T. Baker, 99.8\%) solution at a weight ratio of 2:5:93 ( $\mathrm{ZnO}$ NW : PVDF : NMP). The solution was stirred at $400 \mathrm{rpm}$ for $12 \mathrm{hrs}$ at room temperature to obtain a well-dispersed mixture. The solution was then poured into a plastic container. Subsequently, the composite film was dried for $2 \mathrm{hrs}$ at $65^{\circ} \mathrm{C}$ in order to evaporate the solvent completely. For the uniaxial nanowire composites, the $\mathrm{ZnO}$ nanowires were then aligned by an external electric field at $20 \mathrm{Vpp}$ and $10 \mathrm{MHz}$ by a function generator (4084AWG, BK Precision). Finally, the poling process was conducted at $90^{\circ} \mathrm{C}$ under an electric field of $1 \mathrm{kV} / \mathrm{mm}$.

2.3. Characterization. The morphology of the nanowires was observed using a scanning electron microscope (SEM, FEI Quanta 600). A structural investigation of the $\mathrm{ZnO}$ nanowires was conducted by means of X-ray diffraction (XRD, Bruker-AXS D8 Vario) with $\mathrm{Cu} \mathrm{K} \alpha$ radiation. The frequency-dependent capacitance was determined by an electrochemical workstation (CHI 604D, CH Instruments), and the dielectric constant $\left(\varepsilon_{r}\right)$ was calculated from the measured capacitance.

\section{Results and Discussion}

The alignment process of the nanowires by an external electric field is illustrated in Figure 1. After casting, the nanowires in the composite were randomly oriented in the PVDF matrix. With an alternating electric field, the nanowires were captured in the direction of the electric field and oriented in the same direction by dielectrophoresis (DEP) [20, 21]. Hence, it is possible to align the nanowires along the inplane direction as shown in Figure 1. In the same manner, it is possible to rearrange the nanowires along the out-ofplane direction by applying an external electric field through

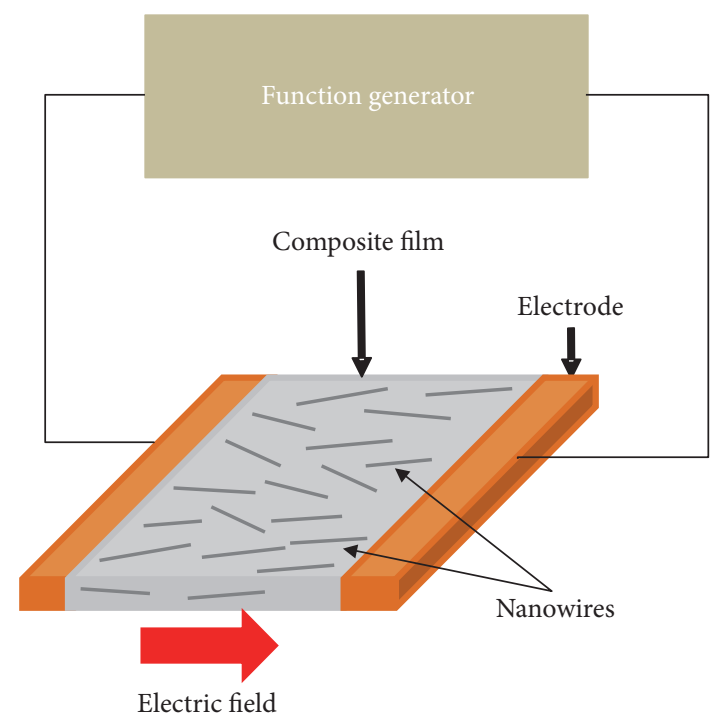

FIGURE 1: Schematic of nanowire alignment by dielectrophoresis in the $\mathrm{ZnO} / \mathrm{PVDF}$ nanocomposite.

the electrodes, which can be installed on the top and bottom of the composite. Such a simple process makes DEP one of the most effective methods by which to manipulate or align nanowires.

Three different sets of composites containing 7.4, 17.5, and $32.3 \mathrm{vol} \%$ of $\mathrm{ZnO}$ nanowires in a PVDF matrix were prepared in order to examine the effect of the nanowire content on the dielectric performance. Even though the concentration of the nanowire was relatively high, the composite film was freestanding, light-weight, and flexible as shown in Figure 2(a). The morphologies of the $\mathrm{ZnO}$ nanowires as obtained by the CVD process are shown in Figure 2(b). The SEM image in the figure shows that the nanowires are suitably grown on the substrate with a densely packed morphology. The diameters of the wires ranged from 75 to $200 \mathrm{~nm}$, and the length ranged from 30 to $45 \mu \mathrm{m}$. The X-ray diffraction of the $\mathrm{ZnO}$ nanowires is shown in Figure 2(c). All deflection peaks in the XRD pattern can be assigned to the hexagonal wurtzite structure of $\mathrm{ZnO}$ with lattice constants of $a=3.249 \AA$ and $c=5.206 \AA$ (space group: P63mc (186)). The clear strong peak pertaining to the $\mathrm{ZnO}$ nanostructure provides evidence of the good crystallinity of the nanowires. Moreover, no reflection peaks from other impurities, such as $\mathrm{ZnCl}_{2}$ or $\mathrm{Zn}$, were detected, which indicates a single-phase hexagonal $\mathrm{ZnO}$ nanostructure without other phases.

All dielectric materials cause polarization by an external electric field or mechanical deformation. The piezoelectric deformation is proportional to the square of the polarization, and the polarization is proportional to the dielectric constant. Therefore, piezoelectric performance can be estimated by a dielectric constant measurement, as more deformation energy would be induced by a larger dielectric constant. In this work, the dielectric constant was obtained by the 


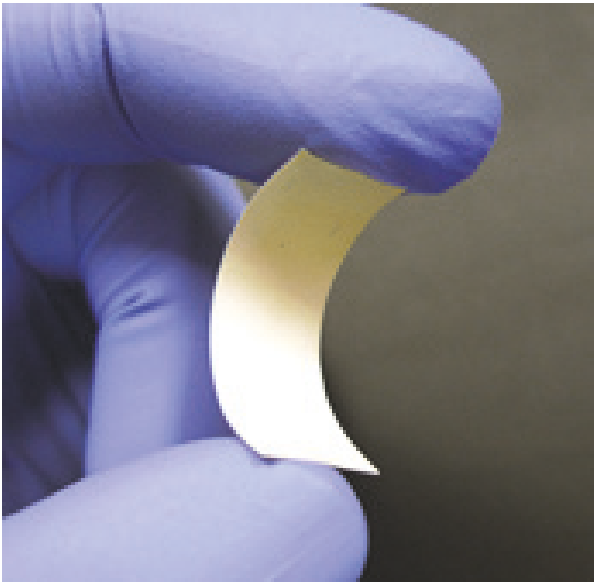

(a)

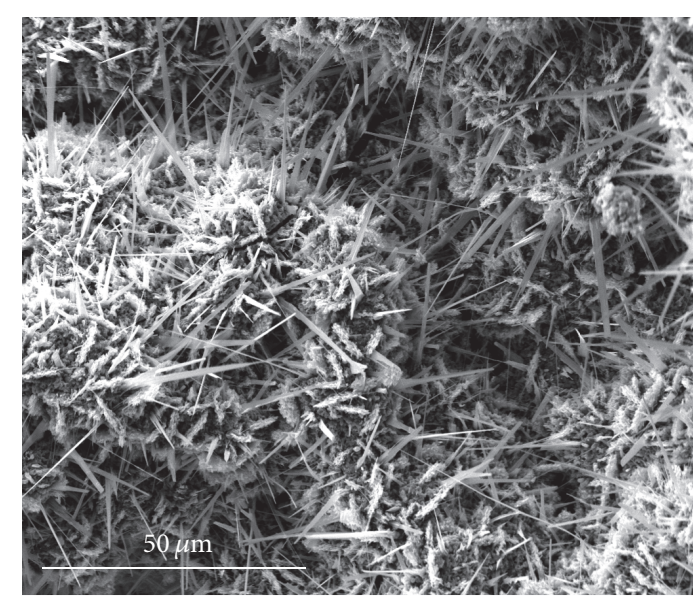

(b)

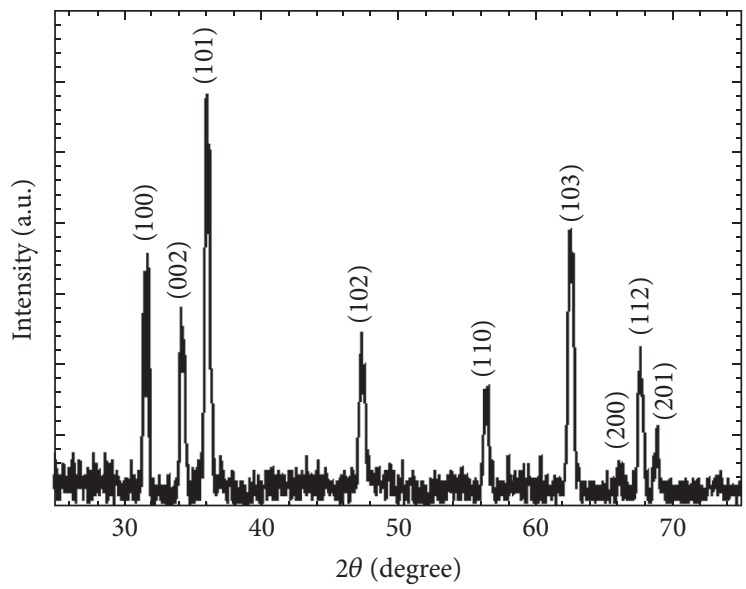

(c)

FIGURE 2: (a) Free-standing and flexible ZnO nanowire filled PVDF composite. (b) SEM and (c) XRD patterns of ZnO nanowires.

AC impedance method [22]. The dielectric constant $\varepsilon_{r}$ is expressed as follows:

$$
\varepsilon_{r}=\frac{C d}{\varepsilon_{o} A}
$$

where $C, \varepsilon_{o}, A$, and $d$ are the capacitance at $1 \mathrm{kHz}$, the free-space dielectric constant $\left(8.854 \times 10^{-12} \mathrm{~F} / \mathrm{m}\right)$, the area $\left(\mathrm{m}^{2}\right)$, and the thickness of the sample $(\mathrm{m})$, respectively. The frequency-dependent capacitance was ascertained by an electrochemical workstation, and the dielectric constant $\left(\varepsilon_{r}\right)$ was then calculated from the measured capacitance with (1). In order to optimize the poling condition, the dielectric constant of the composite containing $7.4 \mathrm{vol} \%$ of $\mathrm{ZnO}$ nanowire was measured by varying the poling voltage. As can be seen in Figure 3, the maximum dielectric constant was obtained as 5.47 at $1 \mathrm{kV} / \mathrm{mm}$ as increasing the poling field, and then breakdown was observed with higher polarization voltage. The relatively low breakdown strength compared to other piezoelectric polymer composites implies that aligned nanowires in the composite induced lower percolation threshold. In Figure 4, the aligned samples before the poling process with $7.4,17.5$, and $32.3 \mathrm{vol} \%$ of the
$\mathrm{ZnO}$ composite show dielectric constants of $2.89,6.30$, and 7.8 , respectively. The results here demonstrate enhancement of 140 200\% compared to the outcomes of randomly oriented and nonpoled samples with identical volume fractions. It is assumed that the piezoelectric performance was improved by high order of the directionality of the aligned nanowires given that this enhancement was observed without a poling process. The increment of nonpoled dielectric constant of composite was reduced with increasing volume fraction of $\mathrm{ZnO}$ nanowires. At 32.3 vol\% of $\mathrm{ZnO}$ nanowire, the dielectric constant was even smaller than that of randomly oriented and poled sample, showing different behavior from the other low concentration samples. This implies that the alignment of nanowires by DEP may not be effective due to the densely packed $\mathrm{ZnO}$ nanowire network structure. However, after the poling process with the aligned samples, the dielectric constant reached 22.8 with $32.3 \mathrm{vol} \%$ of $\mathrm{ZnO}$ nanowires, showing therefore an improvement of $250 \%$ compared to the randomly oriented samples with identical volume fraction of $\mathrm{ZnO}$ nanowires after the poling process. Moreover, the enhancement was $\sim 400 \%$ compared to that of the randomly oriented and nonpoled composite with 


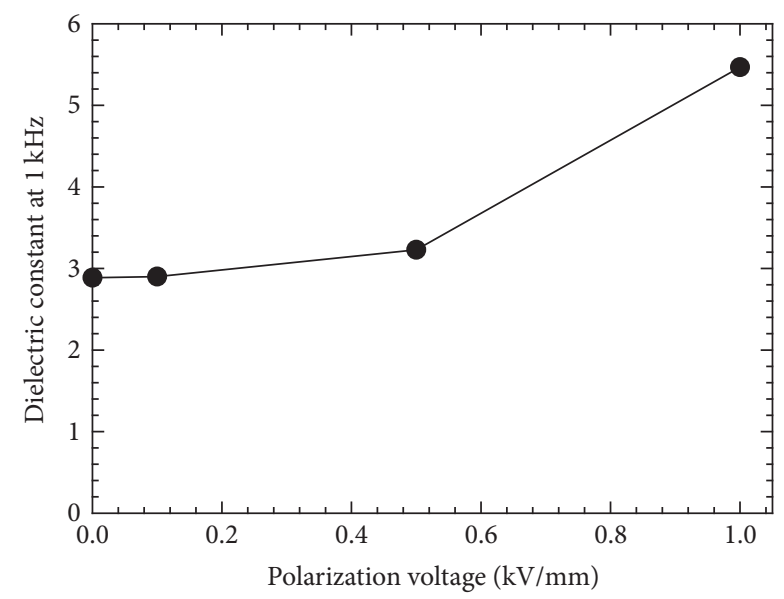

Figure 3: Dielectric constant of the $\mathrm{ZnO} / \mathrm{PVDF}$ composite containing $7.4 \mathrm{vol} \%$ of nanowires after dielectrophoresis as a function of polarization voltage.

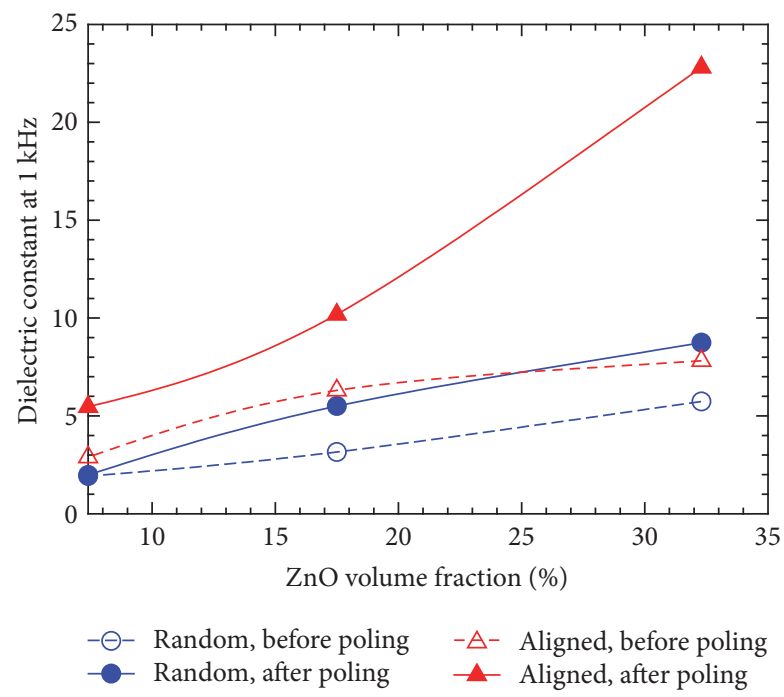

FIgURE 4: Comparison of measured dielectric constant of the $\mathrm{ZnO} / \mathrm{PVDF}$ composites under different condition (random/aligned, before poling/after poling) as a function of $\mathrm{ZnO}$ nanowire volume fraction.

$32.3 \mathrm{vol} \%$ of nanowires. The increment of dielectric constant after poling was enlarged with higher nanowire concentration, which indicates that both of the concentration of the nanowires and the directionality of their network structure are critical factors affecting the piezoelectric performance of the composites. In other words, it can be seen that the poling effect was enhanced by the higher directionality of the fillers which induced reorientation of the randomly distributed single domains.

\section{Conclusion}

In this study, a lead-free piezoelectric polymer composite was introduced with $\mathrm{ZnO}$ nanowires and a PVDF matrix. The $\mathrm{ZnO}$ nanowires were synthesized by the CVD method and different concentrations of nanowires were incorporated into the PVDF matrix. In order to investigate the effect of the directionality of the network structure of the fillers, the nanowires were aligned by DEP. By analyzing the dielectric constant, it was revealed that the effects of the nanowire alignment and the poling process were crucial on the piezoelectric properties of the composite. The dielectric constant of the composite with uniaxial nanowires after the poling process was enhanced by $\sim 400 \%$ compared to that of a randomly oriented nanowire composite without a poling process. In work to extend the findings here, the poling effect can be improved by higher directionality of nanowires with a high aspect ratio. These results suggest a direction to improve the piezoelectric performance of organic composites which can be utilized in lead-free applications such as wearable electronic devices or biosensors.

\section{Conflicts of Interest}

The authors declare that they have no conflicts of interest.

\section{Acknowledgments}

The authors are sincerely grateful for the support from the R\&D Program of Korea Railroad Research Institute, Republic of Korea, and from the 2016 Research Fund of Myongji University.

\section{References}

[1] Q. Lei, Y. Shenfang, W. Qiang, S. Yajie, and Y. Weiwei, "Design and experiment of PZT network-based structural health monitoring scanning system," Chinese Journal of Aeronautics, vol. 22, no. 5, pp. 505-512, 2009.

[2] X. Zhao, H. Gao, G. Zhang et al., "Active health monitoring of an aircraft wing with embedded piezoelectric sensor/actuator network: I. Defect detection, localization and growth monitoring," Smart Materials and Structures, vol. 16, no. 4, article 032, pp. 1208-1217, 2007.

[3] D. A. Van Den Ende, H. J. Van De Wiel, W. A. Groen, and S. Van Der Zwaag, "Direct strain energy harvesting in automobile tires using piezoelectric PZT-polymer composites," Smart Materials and Structures, vol. 21, no. 1, Article ID 015011, 2012.

[4] D. F. Zhou, C. H. Hansen, J. Li, and W. S. Chang, "Review of coupled vibration problems in EMS maglev vehicles," International Journal of Acoustics and Vibration, vol. 15, no. 1, pp. 10-23, 2010.

[5] T. G. King, M. E. Preston, B. J. M. Murphy, and D. S. Cannell, "Piezoelectric ceramic actuators: A review of machinery applications," Precision Engineering, vol. 12, no. 3, pp. 131-136, 1990.

[6] P. K. Panda, "Review: environmental friendly lead-free piezoelectric materials," Journal of Materials Science, vol. 44, no. 19, pp. 5049-5062, 2009.

[7] H. Ohigashi, "Electromechanical properties of polarized polyvinylidene fluoride films as studied by the piezoelectric resonance method," Journal of Applied Physics, vol. 47, no. 3, pp. 949-955, 1976.

[8] E. Pukada, "History and recent progress in piezoelectric polymers," IEEE Transactions on Ultrasonics, Ferroelectrics and Frequency Control, vol. 47, no. 6, pp. 1277-1290, 2000. 
[9] Z. Wang, X. Pan, Y. He, Y. Hu, H. Gu, and Y. Wang, "Piezoelectric nanowires in energy harvesting applications," Advances in Materials Science and Engineering, vol. 2015, Article ID 165631, 21 pages, 2015.

[10] K. Choi, D. Kang, S. Park, and L. Kang, "A study on impact monitoring using a piezoelectric paint sensor," Journal of the Korean Society for Nondestructive Testing, vol. 35, no. 5, pp. 349357, 2015.

[11] W. Choi, K. Choi, G. Yang, J. C. Kim, and C. Yu, "Improving piezoelectric performance of lead-free polymer composites with high aspect ratio $\mathrm{BaTiO}_{3}$ nanowires," Polymer Testing, vol. 53, pp. 143-148, 2016.

[12] Y. Bai, Z.-Y. Cheng, V. Bharti, H. S. Xu, and Q. M. Zhang, "High-dielectric-constant ceramic-powder polymer composites," Applied Physics Letters, vol. 76, no. 25, pp. 3804-3806, 2000.

[13] Z. L. Wang and J. Song, "Piezoelectric nanogenerators based on zinc oxide nanowire arrays," Science, vol. 312, no. 5771, pp. 243246, 2006.

[14] R. Yang, Y. Qin, L. Dai, and Z. L. Wang, "Power generation with laterally packaged piezoelectric fine wires," Nature Nanotechnology, vol. 4, no. 1, pp. 34-39, 2009.

[15] H. Tang, Y. Lin, and H. A. Sodano, "Enhanced energy storage in nanocomposite capacitors through aligned PZT nanowires by uniaxial strain assembly," Advanced Energy Materials, vol. 2, no. 4, pp. 469-476, 2012.

[16] P. X. Gao, J. Song, J. Liu, and Z. L. Wang, "Nanowire piezoelectric nanogenerators on plastic substrates as flexible power sources for nanodevices," Advanced Materials, vol. 19, no. 1, pp. 67-72, 2007.

[17] N. Mishra, B. Krishna, R. Singh, and K. Das, "Evaluation of Effective Elastic, Piezoelectric, and Dielectric Properties of SU8/ZnO Nanocomposite for Vertically Integrated Nanogenerators Using Finite Element Method," Journal of Nanomaterials, vol. 2017, pp. 1-14, 2017.

[18] E. S. Nour, M. O. Sandberg, M. Willander, and O. Nur, "Handwriting enabled harvested piezoelectric power using $\mathrm{ZnO}$ nanowires/polymer composite on paper substrate," Nano Energy, vol. 9, pp. 221-228, 2014.

[19] G. Zheng, F. Patolsky, Y. Cui, W. U. Wang, and C. M. Lieber, "Multiplexed electrical detection of cancer markers with nanowire sensor arrays," Nature Biotechnology, vol. 23, no. 10, pp. 1294-1301, 2005.

[20] A. W. Maijenburg, M. G. Maas, E. J. B. Rodijk et al., "Dielectrophoretic alignment of metal and metal oxide nanowires and nanotubes: a universal set of parameters for bridging prepatterned microelectrodes," Journal of Colloid and Interface Science, vol. 355, no. 2, pp. 486-493, 2011.

[21] C. S. Lao, J. Liu, P. Gao et al., " ZnO nanobelt/nanowire schottky diodes formed by dielectrophoresis alignment across au electrodes," Nano Letters, vol. 6, no. 2, pp. 263-266, 2006.

[22] H. Tang, Y. Lin, C. Andrews, and H. A. Sodano, "Nanocomposites with increased energy density through high aspect ratio PZT nanowires," Nanotechnology, vol. 22, no. 1, Article ID 015702, 2011. 

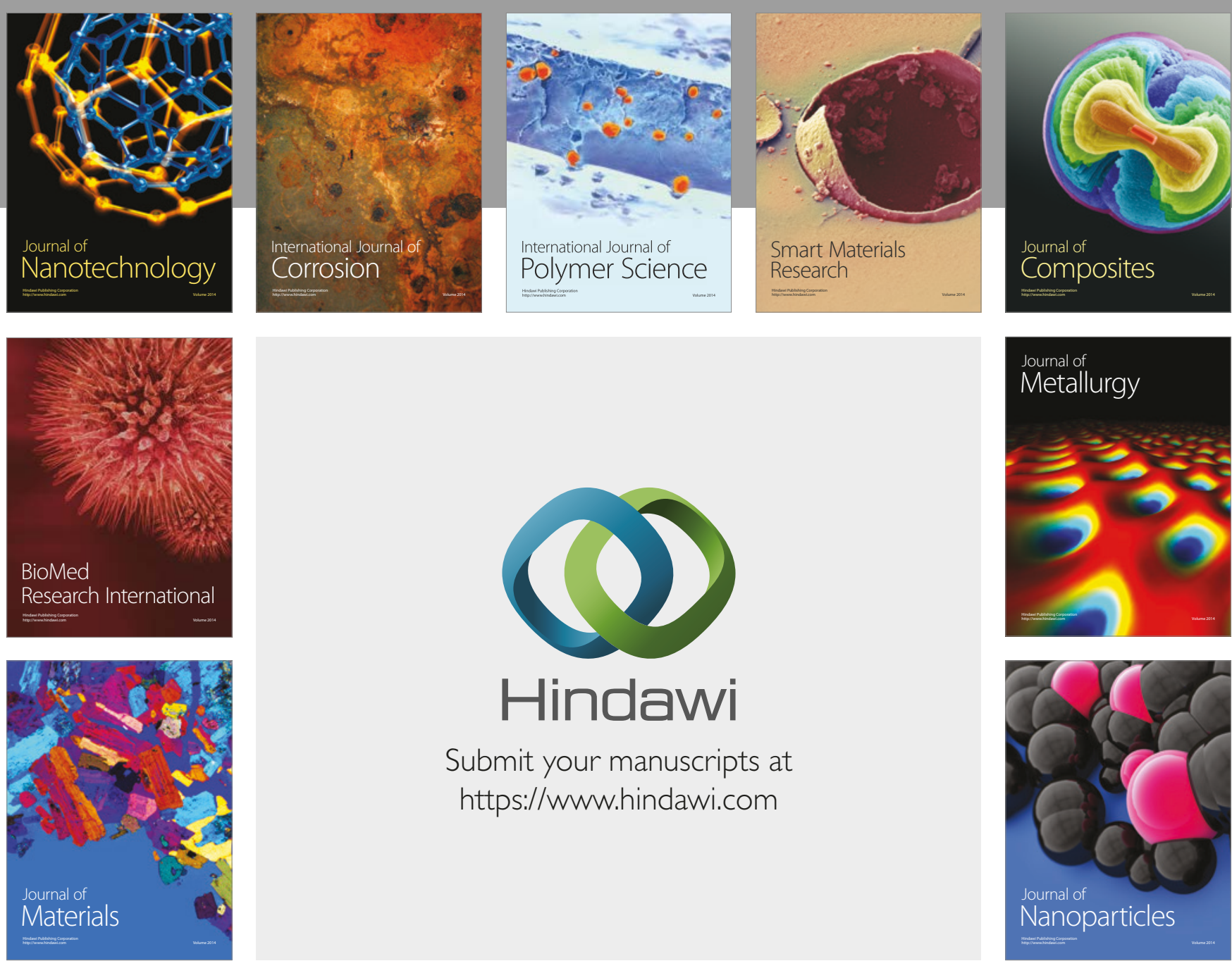

\section{Hindawi}

Submit your manuscripts at

https://www.hindawi.com
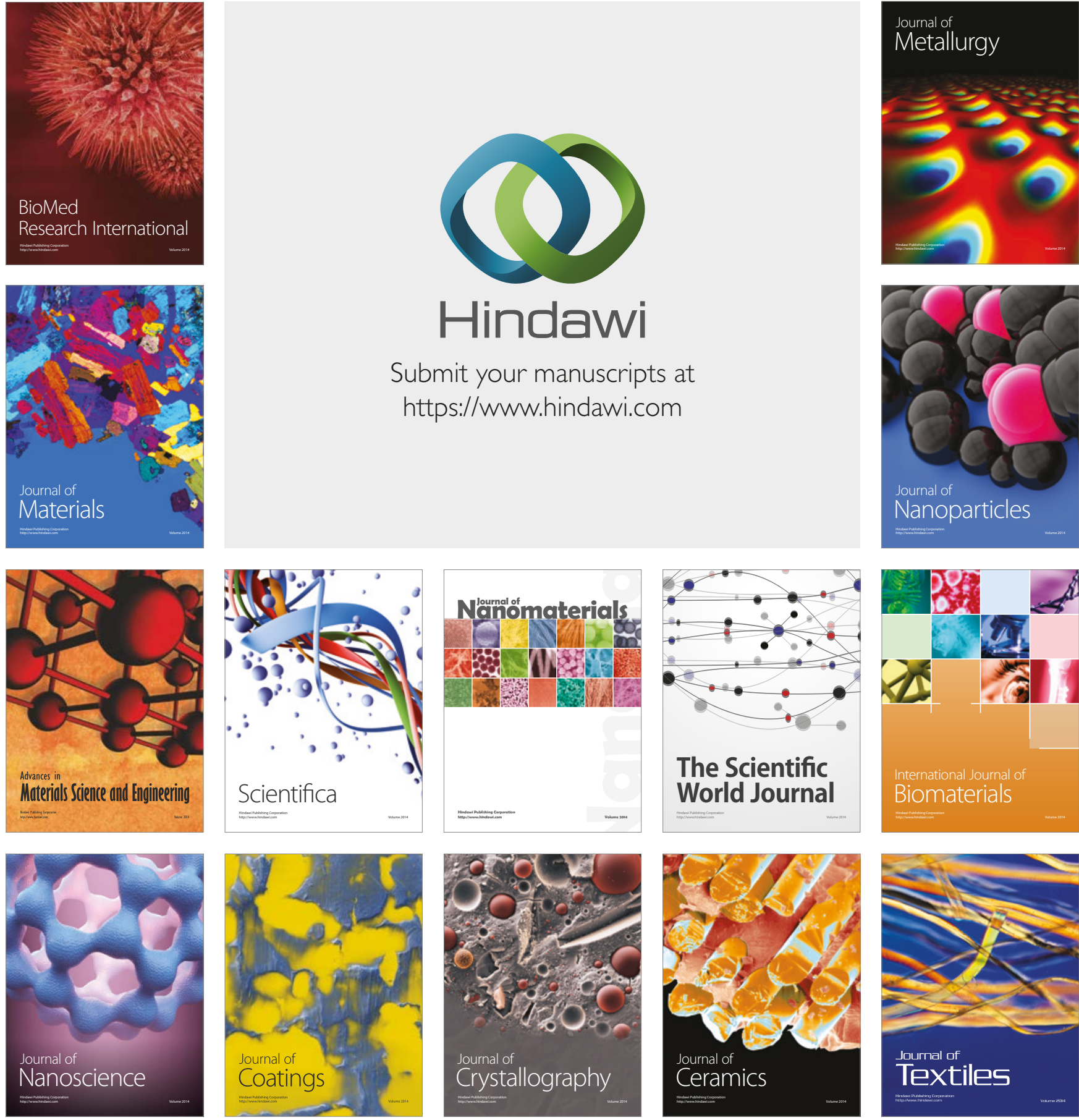

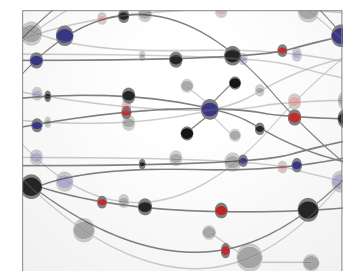

The Scientific World Journal
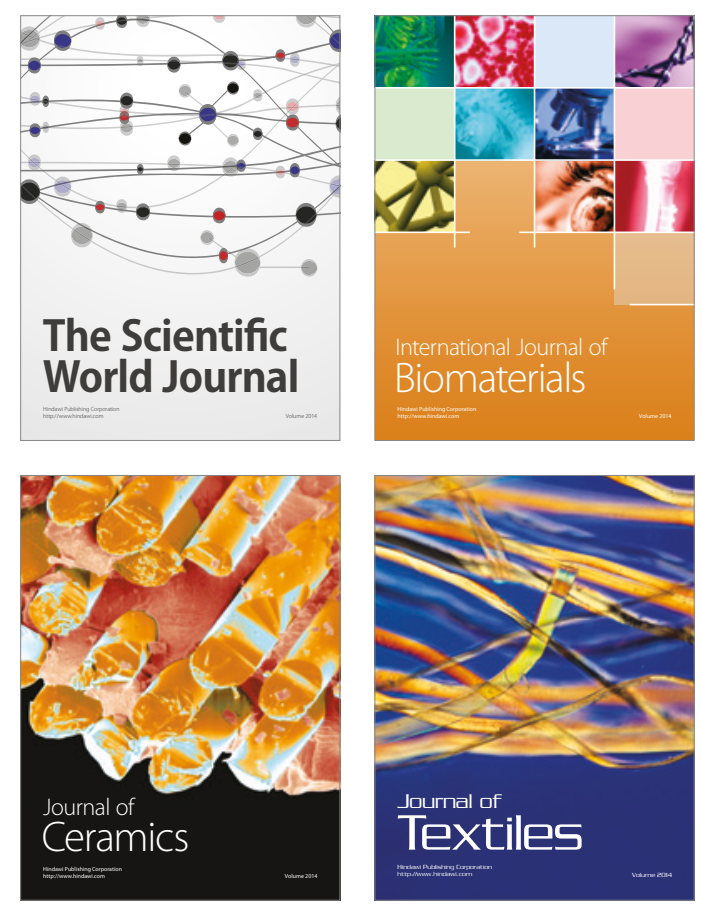